\title{
Basilar artery fenestration associated with aneurysms treated by endovascular approach
}

\author{
Stefanita Dima ${ }^{1}$, Mugurel Radoi $^{2}$ \\ "Carol Davila" University of Medicine and Pharmacy, Bucharest \\ National Institute of Neurology and Neurovascular Diseases \\ ${ }^{1}$ Neuroradiological Department; ${ }^{2}$ Neurosurgical Department
}

\begin{abstract}
Introduction: Arterial fenestrations are associated with saccular aneurysms that are often difficult to treat with open surgical techniques. Basilar artery fenestration reported in the literature is highly variable depending on the technique used. Typically fenestration occurs at the lower end of the basilar artery just at the vertebral arteries join. For basilar artery fenestrations associated with aneurysms endovascular embolization could be the first treatment choice.
\end{abstract}

Methods: This study presented three cases of patients having basilar artery fenestration associated with aneurysm that were treated endovascularly. All patients underwent endovascular embolization by femoral approach, under general anesthesia.

Results: In all three cases, no new neurological deficits were reported. Balloon remodeling technique was necessary in one patient that presented kissing aneurysms. The length of the follow-up was 3 years for 2 patients, and 1 year for one patient. All the aneurysms, except one, presented a small recanalization at four vessels digital subtraction angiography (DSA) control, but it remained stable even at the three years control.

Conclusions: Endovascular treatment of basilar artery aneurysms associated with fenestrations is a safe and durable option. No second embolization procedure was necessary in our cases. No limb of the fenestration was necessary to be sacrificed. Larger series of patients treated with this method are needed to support our evidence.

Key words: aneurysms, basilar artery fenestration, endovascular embolization, three years follow-up.

\section{Introduction}

Basilar artery fenestration is the most common intracranial arterial fenestration. The term "fenestration" refers to a localized duplication of a vessel. Its reported in the literature is highly variable depending on the technique used:

- $0.3-0.6 \%$ at four vessels digital subtraction angiography (presumably low, due to preferential laminar flow in only one lumen at a time) $(9,11)$.

- $2 \%$ on magnetic resonance angiography (12)

- up to $5.26 \%$ in autopsy series (11).

Typically, fenestration occurs at the lower end of the basilar artery, just at the vertebral arteries join. However, it can also be seen in the mid- and distal portion (tip) of the basilar artery $(2,5)$.

The basilar artery is formed by fusion of the plexiform primitive longitudinal neural 
arteries in a craniocaudal direction by approximately the 5 th fetal week (8). If these embryonic precursors fail to fuse completely, duplication or fenestration of the basilar artery results (10). There is an increased incidence of basilar aneurysm formation at the site of fenestration, presumably due to abnormal flow dynamics (3). They are reported in $7 \%$ of fenestrations (6). Medial defects, a common feature in both brain arteries and fenestrations, may predispose the arterial fenestration to aneurysm formation (4).

The aim of this study is to present three rare cases of basilar arterial fenestration associated with aneurysm and also to propose the endovascular treatment as the first therapeutic choice for these particular fenestration aneurysms.

\section{Material and methods}

We reported the cases of three patients, with subarachnoid hemorrhage, who were diagnosed by four vessels digital subtraction angiography with basilar artery fenestration associated with aneurysm. Treatment decision (clip or coil) was based on an interdisciplinary approach - cerebrovascular neurosurgeon and neuroradiologist favouring the treatment modality associated with the lowest periprocedural risk and the highest anticipated rate of aneurysm occlusion. The group of three patients presented 4 aneurysms and they were treated on the endovascular route with coils. All patients underwent endovascular embolization by the femoral route, under general anesthesia.

\section{First case}

A 42 years old female was admitted to the hospital with terrible headache, nausea, neck stiffness and sensitivity to light. No focal neurological deficits were documented. Cerebral computed tomography showed a subarachnoid hemorrhage, located mainly in the posterior fosa. Four vessels digital subtraction angiography demonstrated a basilar artery fenestration associated with an aneurysm at the site of fenestration figure 1 (1a, 1b, 1c). The endovascular embolization with coils was performed 5 days after diagnosis, and the immediate control cerebral angiography showed complete occlusion of the aneurysm figure $1(1 \mathrm{~d}, 1 \mathrm{e})$. The patient left the hospital in good neurological state, 5 days after embolization. Regularly followup (yearly) was performed using DSA. Cerebral control DSA at 37 months after embolization demonstrated a very small recanalization of the aneurysm neck (Raymond I), but the aneurysm was stable and no further embolization was considered to be necessary figure 1 (1f).

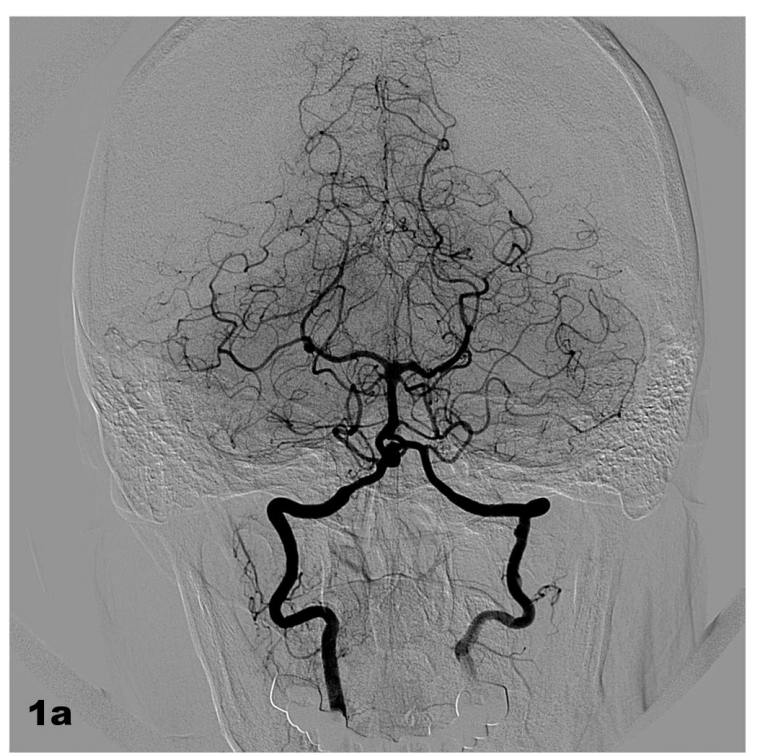



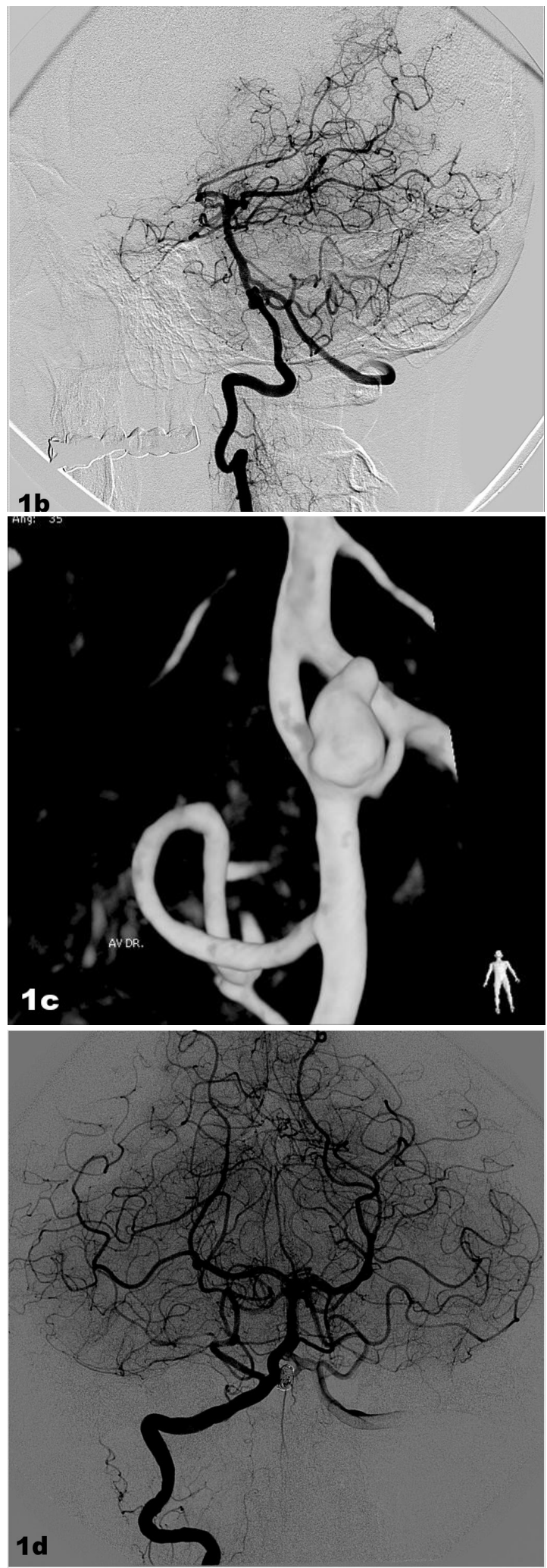

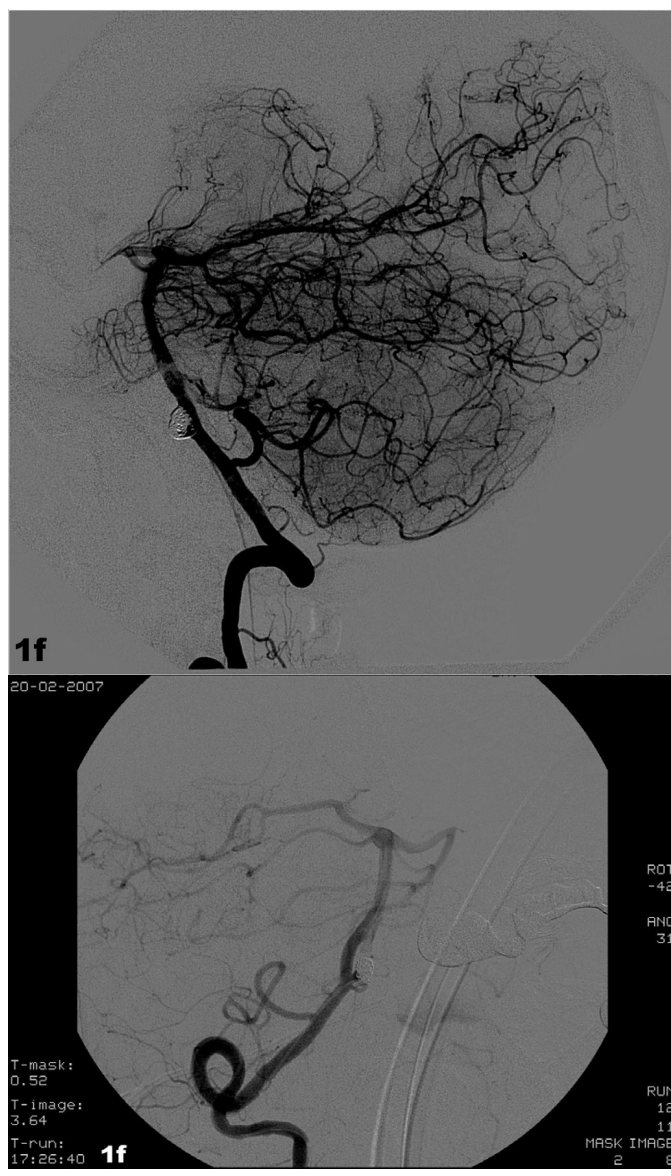

Figure 1 A, B,C - Cerebral digital subtraction angiography of the basilar artery injected from the right vertebral artery showed a basilar artery fenestration associated with an aneurysm: 2D images (1a, 1b), 3D image (1c); 1d, 1e - Cerebral DSA of the basilar artery at the end of the embolization procedure, showed that coils filled completely the aneurysm; 1f - Cerebral control DSA at 37 months demonstrated a very small recanalization of the aneurysm neck.

\section{Second case}

A 45 years old male was admitted in our clinic presented similar symptoms that the previous case. He was alert and oriented (GCS-15 pct.), and his cerebral computed tomography showed a subarachnoid hemorrhage. The DSA showed the same basilar artery fenestration associated with an aneurysm figure $2(2 \mathrm{a}, 2 \mathrm{~b})$. The decision was to treat the aneurysm by coils 
embolization, which lead to a complete occlusion of the aneurysm figure $2(2 \mathrm{c}, 2 \mathrm{~d})$. The patient was discharged with no focal neurological deficits 3 days after embolization. The cerebral DSA follow-up demonstrated, at 24 months after embolization, a very small recanalization of the aneurysm neck (Raymond I) figure 2 (2e, 2f). Because the aneurysm was stable, no further embolization was considered to be necessary at that time, and a new cerebral DSA control was planed to be performed after one year.
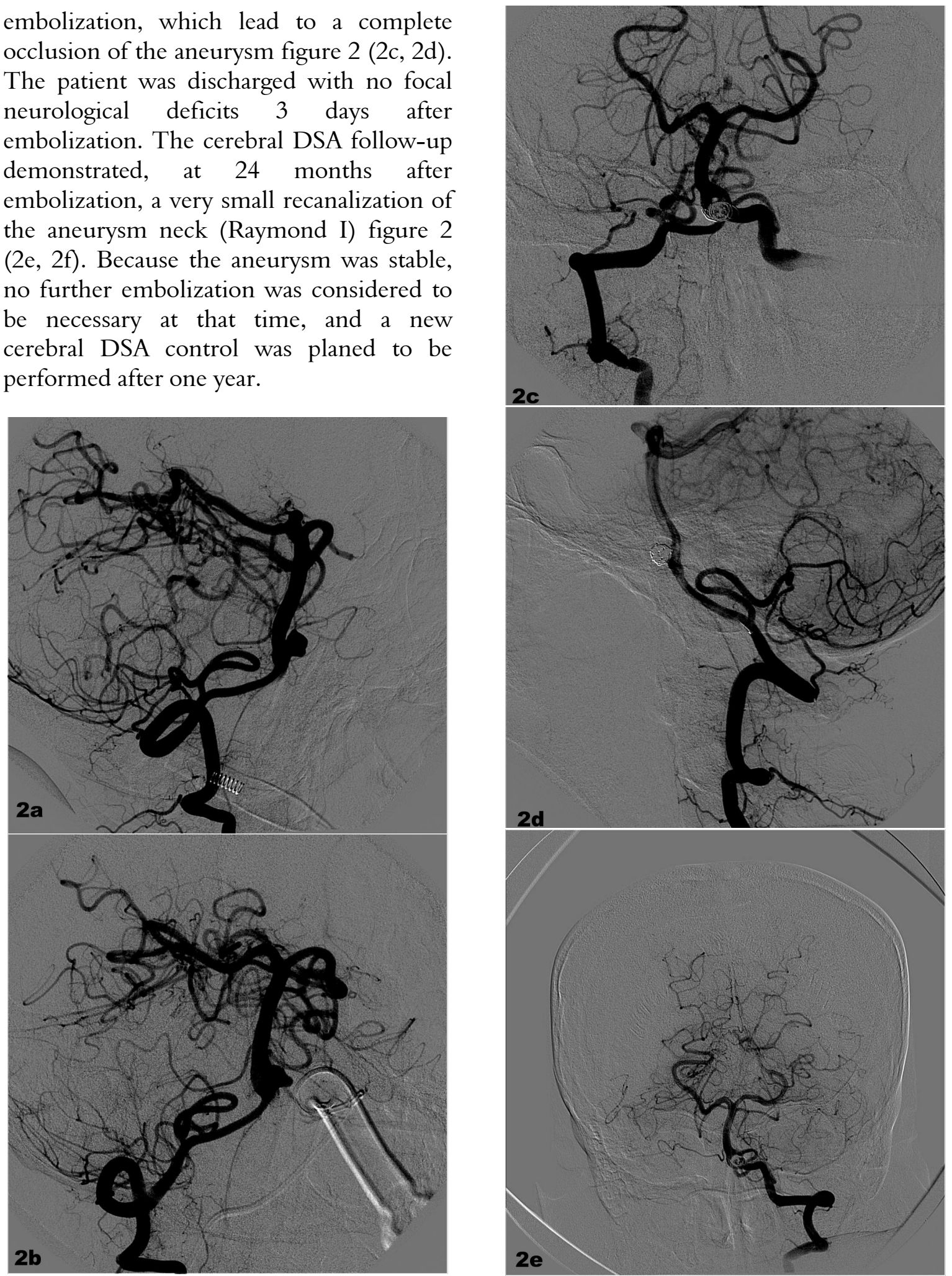


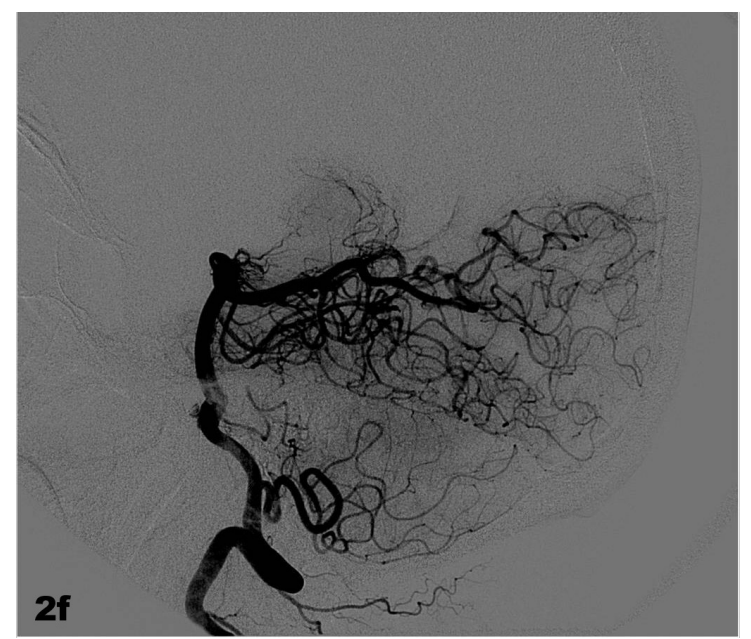

Figure 2 A, B - Cerebral digital subtraction angiography of the basilar artery injected from the right vertebral artery demonstrated the aneurysm and the fenestration of the basilar artery; $2 \mathrm{c}, 2 \mathrm{~d}-$ Cerebral DSA performed after embolization procedure demonstrated complete occlusion of the aneurysm; 2e, $2 \mathrm{f}$ - Cerebral control DSA demonstrated, at 24 months ,a very small recanalization of the aneurysm neck.

\section{Third case}

A 41 years old female came to the hospital complaining of terrible headache, neck stiffness, sensitivity to light and vomiting. She showed no focal neurological deficits. Cerebral computed tomography presented subarachnoid hemorrhage and a cerebral DSA was performed. It showed a basilar artery aneurysm associated with two aneurysms at the site of fenestration (kissing aneurysms) figure 3 ( $3 a, 3 b, 3 c)$. Both aneurysms were treated by coils embolization figure 3 (3d, 3e). Four days later, the patient was discharged in a good neurological state. The cerebral DSA follow-up demonstrated at 12 months after coils embolization, that one of the aneurysm presented a small recanalization of its neck figure 3 (3f, $3 \mathrm{~g}$ ). The decision was to continue yearly follow-up, in order to notice if the recanalization will grow or remain stable.
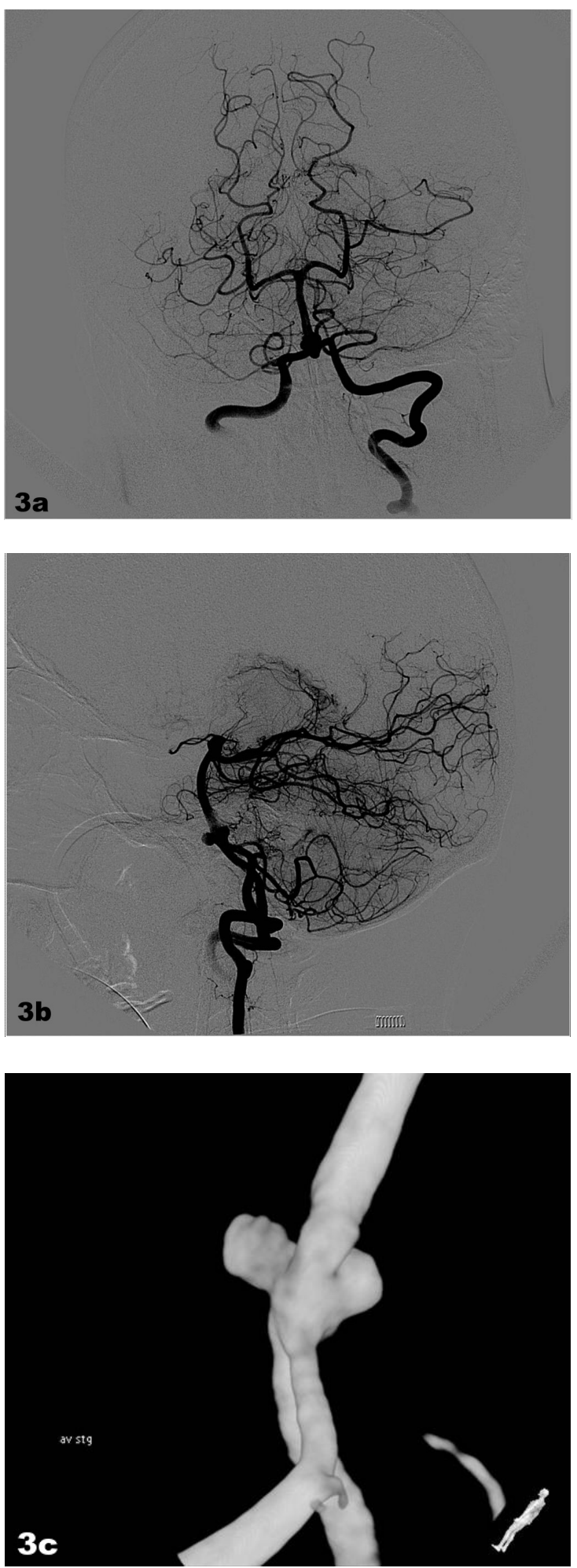

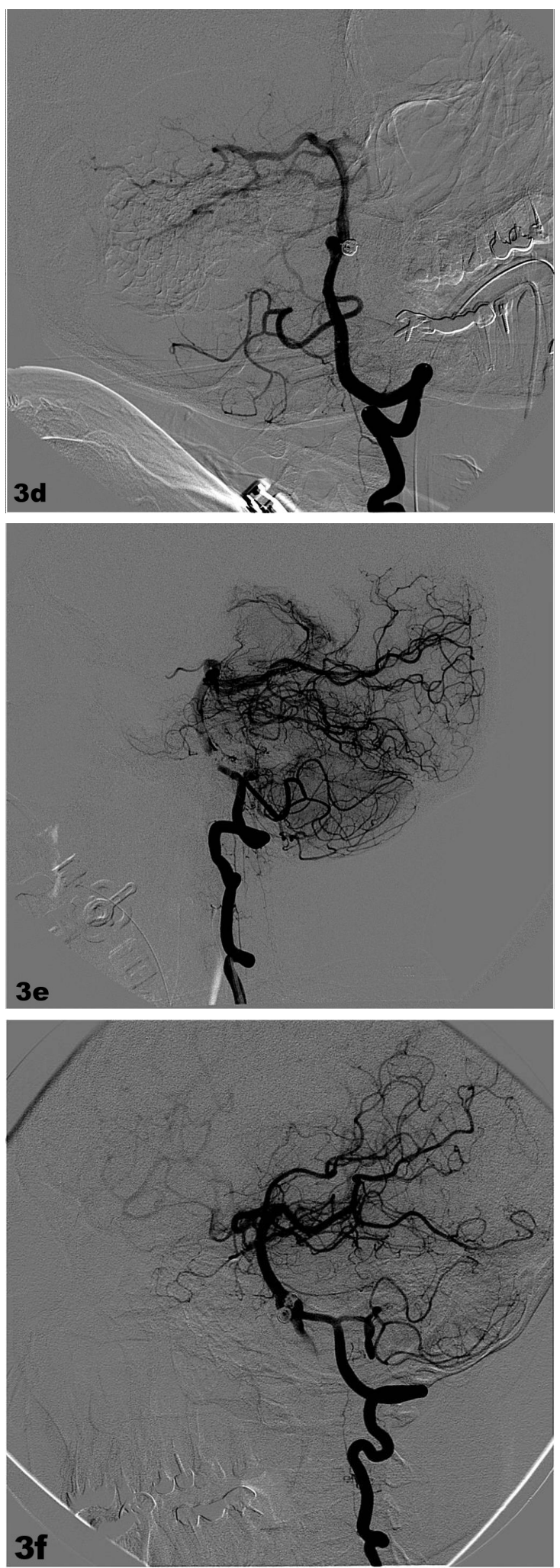

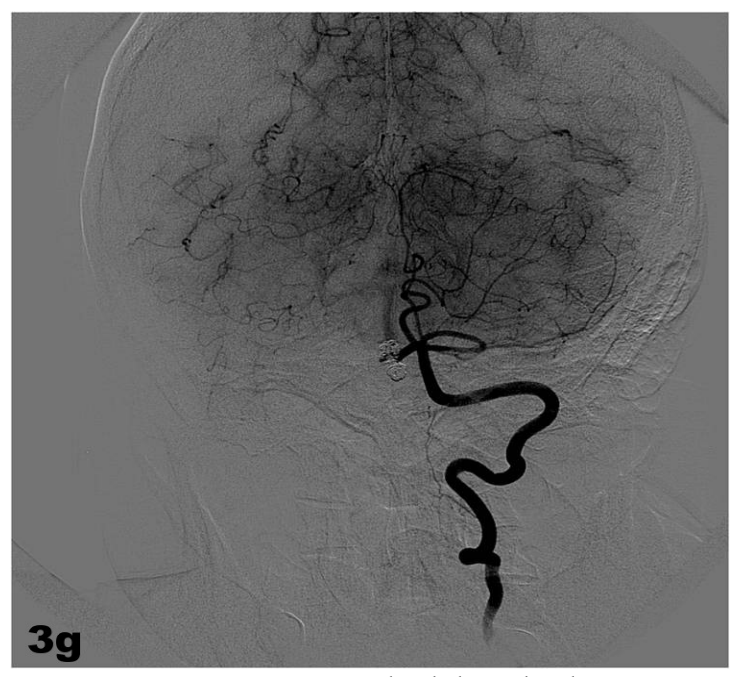

Figure 3 A, B, C - Cerebral digital subtraction angiography of the basilar artery injected from the right vertebral artery showed kissing aneurysms and the fenestration at the lower end of the basilar artery: 2D images (3a, 3b), 3D image (3c); 3d, 3e - Cerebral

DSA after embolization procedure demonstrated complete occlusion of the aneurysms; $3 \mathrm{f}, 3 \mathrm{~g}$ Control cerebral DSA demonstrated, at 12 months after coils embolization, that one of the aneurysm presented a small recanalization of its neck.

\section{Results}

The clinical outcome was very good for all patients. There were no embolizationrelated complications. Admission status of the patients was good and no new focal neurological deficits were encountered. All the aneurysms, except one, had a small recanalization at the first control cerebral DSA, which was performed for all patients, at 3 month after embolization procedure. But this recanalization of the aneurysm neck remain stable even at three years control, so no second embolization procedure was necessary.

\section{Discussion}

Basilar artery surgery is related to some obvious technical difficulties because of size, configuration or location of the aneurysm. Treatment of vertebrobasilar junction or lower trunk basilar aneurysms continues to advance with improvements in 
DOI: $10.2478 / v 10282-012-0015-0$

microsurgical technique, skull base approaches and advances in endovascular treatment. Good clinical outcomes in shortterm appear to be possible with endovascular detachable coil embolization in this particular aneurysms location $(1,8)$.

The largest series in the literature of aneurysms developed at a basilar artery fenestration, which were treated by endovascular embolization, was published by a Islak C and co-workers in 2002 (7). Between November 1994 and February 2000 , by means of endovascular detachable coils, they treated 10 consecutive patients with 11 basilar artery aneurysms associated with fenestrations. In their series, initial complete occlusion of the aneurysm was achieved in 10 of the 11 aneurysms. There was only one re-growth at 1-year followup, which was successfully treated again. Four of the aneurysms were treated initially with balloon remodeling. In one case, one limb of the fenestration was sacrificed (7).

In our series of 3 patients, with 4 aneurysms, balloon remodeling technique was used only in the patients who presented two kissing aneurysms. No limb of the fenestration was sacrificed. On cerebral DSA follow-up, a small recanalization of the aneurysm neck was noticed in 3 of the 4 aneurysms that were filled with coils. In all cases, aneurysms were stable, without signs of re-growing or subarachnoid hemorrhage, so a second embolization was not considered to be necessary. For the first patient, because the recanalization did not change in size, and the aneurysm was stable, the next planed cerebral DSA will be in 10 years. For the other 2 patients, 2 consecutive yearly cerebral DSA will be necessary to correctly assess the size or, eventually, the change in size of the recanalization. If this will remain stable, the same attitude as for the first patient will be adopted.

\section{Conclusion}

Treatment of these difficult lesions still is associated with high management morbidity and mortality, however, relative to anterior circulation aneurysms. Endovascular treatment of basilar artery aneurysms associated with fenestrations appears to offer advantages over traditional open surgical techniques, and is a safe and durable therapeutic option. Larger series of patients treated with this method are needed to support our evidence.

\section{References}

1. Bavinski G, Killer M, Gruber A (1999). Treatment of basilar bifurcation aneurysms by using Guglielmi detachable coils: A 6-years experience. J Neurosurg 90:843-852.

2. Danaila L, Stefanescu Fl. (2007). Anevrisme cerebrale. (pp 652-665). Bucuresti. Ed. Academiei Romane.

3. De Caro R, Serafini MT, Galli S, Parenti A, Guidolin D, Munari PF (1995). Anatomy of segmental duplication in the human basilar artery: possible site of aneurysm formation. Clin Neuropathol 14:303-309.

4. Finlay HM, Canham PB (1994). The layered fabric of cerebral artery fenestrations. Stroke 25(9):1799-1806.

5. Fujimura M, Sugawara T, Higuchi H, Oku T, Seki H (1997). A ruptured aneurysm at the distal end of the basilar artery fenestration associated with multiple fenestrations of the vertebrobasilar system: case report. Surg Neurol 47:469-472.

6. Imaizumi T, Saito K, Kobayashi T, Sakamoto Y, Komeichi T (1996). Saccular aneurysm associated with fenestration of the distal segment of basilar artery [in Japanese]. No Shinkei Geka 24:639-642.

7. Islak C., Kocer N., Kantarci F., Saatci I., Uzma O., Canbaz B (2002). Endovascular management of basilar artery aneurysms associated with fenestrations. AJNR 23: 958-964.

8. Nagashima H, Okudera H, Orz Y, Nakagawa F (1999). Endovascular treatment of basilar trunk aneurysm associated with fenestration of the basilar artery. Neurosurg Rev 22:219 -221.

9. Osborn GA (1999). Diagnostic Cerebral Angiography (pp. 190-193). Philadelphia, Lippincott Williams \& Wilkins.

10.Padget DH (1948). The development of the cranial arteries in the human embryo. Contrib Embryol 32:205-261.

11.Sanders WP, Sorek PA, Mehta BA (1993). Fenestration of intracranial arteries with special attention to associated aneurysms and other anomalies. AJNR Am J Neuroradiol 14:675-680.

12. Uchino A, Kato A, Takase Y, Kudo S (2001). Basilar artery fenestrations detected by MR angiography. Radiat Med 19:71-74. 